\title{
Crossover between the Adiabatic and Nonadiabatic Limits of Thermal Electron Transfer: Verifying the Landau-Zener Formula
}

Guang Yuan Zhu

Jinan University

Yi Qin

Jinan University

Miao Meng

Jinan University

Suman Mallick

Jinan University

Hang Gao

Jinan University

Xiaoli Chen

Jinan University

Tao Cheng

Jinan University

Ying Ning Tan

Jinan University

Xuan Xiao

Jinan University

Mei Juan Han

Jinan University

Meifang Sun

Jinan University

Chun Yuan Liu ( $\nabla$ tcyliu@jnu.edu.cn )

Jinan University https://orcid.org/0000-0001-6908-9929

Article

Keywords: electron transfer, ET, adiabatic and nonadiabatic limits, contemporary ET theories

Posted Date: August 6th, 2020

DOl: https://doi.org/10.21203/rs.3.rs-46642/v1 
License: (c) (i) This work is licensed under a Creative Commons Attribution 4.0 International License. Read Full License

Version of Record: A version of this preprint was published at Nature Communications on January 19th, 2021. See the published version at https://doi.org/10.1038/s41467-020-20557-7. 


\section{Crossover between the Adiabatic and Nonadiabatic Limits of Thermal Electron}

\section{Transfer: Verifying the Landau-Zener Formula}

Guang Yuan Zhu, ${ }^{\dagger}$ Yi Qin, ${ }^{\dagger}$ Miao Meng, ${ }^{\dagger}$ Suman Mallick, Hang Gao, Xiaoli Chen, Tao Cheng, Ying Ning Tan, Xuan Xiao, Mei Juan Han, Meifang Sun and Chun Y. Liu*

${ }^{1}$ Department of Chemistry, Jinan University, 601 Huang-Pu Avenue West, Guangzhou 510632, China

The semiclassical models of nonadiabatic transition were proposed first by Landau and Zener in 1932, which has been widely used in study of electron transfer (ET); however, experimental substantiation of the Landau-Zener formula remains challenging. Herein, employing the Marcus-Hush theory, thermal ET in mixed-valence complexes $\left\{\left[\mathrm{Mo}_{2}\right]-(\mathrm{ph})_{n^{-}}\right.$ $\left.\left[\mathrm{Mo}_{2}\right]\right\}^{+}(n=1-3)$ is investigated and the Landau-Zener analysis performed in the adiabatic and nonadiabatic limits. Evidently, the Landau-Zener formula is valid in the adiabatic regime in a broader range of conditions than the theoretical limitation known as the narrow avoided-crossing. The intermediate system is identified with an overall transition probability $\left(\kappa_{\mathrm{el}}\right)$ of $\sim 0.5$, which is resolved by the contributions from the single and the first multiple passage. The results unify the contemporary ET theories under the semiclassical framework. The obtained insights help to understand and control the ET processes in biological and chemical systems.

Electron transfer (ET) is a long-standing research subject in chemistry. ${ }^{1,2,3,4}$ The study leads to better understanding of charge transport in physics and materials science and the enzymatic redox processes in biology ${ }^{5,6}$ and thus, supports the development of modern technologies including 
molecular electronics ${ }^{7}$ and solar energy conversion. ${ }^{8}$ According to the Marcus theory, ${ }^{1,3,5}$ ET rate is governed by three physical parameters: the Gibbs free energy change $\left(\Delta G^{\circ}\right)$, the reorganization energy $(\lambda)$ and the electronic coupling (EC) matrix element $\left(H_{\mathrm{ab}}\right)$. Quantities $\lambda$ and $H_{\mathrm{ab}}$ are the nuclear and electronic factors, respectively, which affect ET process due to the differences in time scales of nuclear vibrations and electron transmission. Both intramolecular and intermolecular ET reactions may occur adiabatically and nonadiabatically, depending on the interplay of the atomic and electronic dynamics of the system and medium. Comparison between the electron hopping frequency $\left(v_{\mathrm{el}}\right)$ and nuclear vibrational frequency $\left(v_{\mathrm{n}}\right)$ determines ET in the two regimes, that is, 2,9 adiabatic: $v_{\mathrm{el}}>>v_{\mathrm{n}}$; nonadiabatic: $v_{\mathrm{el}}<<v_{\mathrm{n}}$

10 Nonadiabatic transition of reactions from reactant to product was described first by Landau and Zener in 1930s. ${ }^{10,11,12}$ The semiclassical Landau-Zener (LZ) model discriminates quantitatively the nonadiabatic and adiabatic limits by three parameters: adiabatic parameter $\gamma$ (eq 1a) nonadiabatic transition probability $P_{0}(\mathrm{eq} 1 \mathrm{~b})$ and electronic transmission coefficient $\kappa_{\mathrm{el}}(\mathrm{eq}$ $1 \mathrm{c}),{ }^{1,2,13}$

$$
\begin{aligned}
\gamma & =\frac{H_{a b}^{2}}{2 h v_{n}} \sqrt{\frac{\pi}{\lambda k_{\mathrm{B}} T}} \\
P_{0} & =1-\exp (-2 \pi \gamma) \\
\kappa_{\mathrm{el}} & =2 P_{0} /\left(1+P_{0}\right)
\end{aligned}
$$

When $\gamma \gg>1$, the adiabatic limit is realized and for thermal ET $\kappa_{\mathrm{el}} \approx 1$, while the nonadiabatic limit prevails with $\gamma<<1 .{ }^{1,2,13}$ By definition of $\gamma$ (eq 1a), it is clear that nonadiabatic transition depends upon the electronic and nuclear factors, represented by $H_{\mathrm{ab}}$ and $v_{\mathrm{n}}$, respectively. According the LZ model, thermal ET through nonadiabatic transition takes place in the vicinity of the conical area when the adiabatic avoided crossing is similar to the diabatic crossing. This brings up the general condition $\Delta G^{*}>>H_{\mathrm{ab}}{ }^{2,12}$

The LZ formula has been exploited to predict whether an ET reaction is adiabatic or nonadiabatic; however, experimental validation of the theory itself has not been achieved. Moreover, identification of the intermediate between the two limits and elucidation of system transformation from one to the other limit are nontrivial, which have been actively explored by theoreticians. ${ }^{2,12,14,15,16}$ Up to now, no experimental study describes the energetic and dynamic features of the transient intermediate. Verifying the LZ model and characterizing the intermediate 
can be possibly accomplished in elemental ET reactions, if an array of systems with the electronic dynamics spanning a broad range of time scales with respect to the nuclei is developed. Photoinduced ET is generally in the nonadiabatic regime, while thermal ET occurs usually adiabatically with $v_{\mathrm{el}} \gg v_{\mathrm{n}}$. Testing the LZ model (eq 1) also encounters the technique problems. ${ }^{15}$ For example, time-resolved spectroscopy ${ }^{3}$ and spectral line-broadening analysis ${ }^{17}$ are not appropriate because these methodologies do not provide independent coupling integral $\left(H_{\mathrm{ab}}\right)$ and kinetic parameters as required. Mixed-valence (MV) complexes with two bridged redox sites, generally denoted as a $\mathrm{D}$ (donor)-B(bridge)-A(acceptor) assembling, are favorable experimental models due to the properties of the intervalence charge transfer (IVCT) absorption which measures directly the Frank-Condon barrier of ET $\left(E_{\mathrm{IT}}\right)$ between the electron donor and acceptor, ${ }^{18}$ which equals to the reorganization energy $(\lambda)$ for symmetrical system $\left(\Delta \mathrm{G}^{\circ}=0\right)$ based on $E_{\mathrm{IT}}=\Delta \mathrm{G}^{\circ}+$

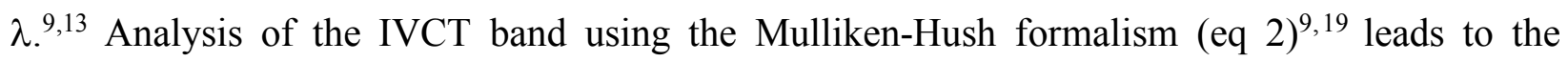
coupling energy. ${ }^{20}$

$$
H_{\mathrm{ab}}=\frac{2.06 \times 10^{-2}}{r_{\mathrm{ab}}}\left(\varepsilon_{\mathrm{IT}} \Delta v_{1 / 2} E_{\mathrm{IT}}\right)^{1 / 2}
$$

This $H_{\mathrm{ab}}$ parameter can be used to calculate the electronic transmission frequency ( $\left.v_{\mathrm{el}}\right)$ (eq 3$)$, the adiabatic parameter $(\gamma)$ (eq 1a) and the optical or the thermal ET kinetics based on semiclassical theory at the high temperature limit. 5,21

$$
v_{\mathrm{el}}=\frac{2 H_{\mathrm{ab}}^{2}}{h} \sqrt{\frac{\pi^{3}}{\lambda k_{\mathrm{B}} T}}
$$

This approach was first proposed by Taube in 1986; ${ }^{22}$ unfortunately, it has not succeeded for many decades. The reason for this is that few MV molecular systems exhibit characteristic IVCT bands that allow optical derivations of the ET dynamics and kinetics, ${ }^{9,23,24}$ although many efforts have been devoted since the Cruetz-Taube ion $\left[\left(\mathrm{NH}_{3}\right)_{5} \mathrm{Ru}(\mathrm{pz}) \mathrm{Ru}\left(\mathrm{NH}_{3}\right)_{5}\right]^{5+}$ was synthesized in 1970s. ${ }^{18,22,25}$

Given the characteristic IVCT bands, mixed-valence D-B-A molecular systems with a quadruply-bonded $\mathrm{Mo}_{2}$ unit ${ }^{26}$ as the donor and a $\mathrm{Mo}_{2}$ unit having a bond order 3.5 as the acceptor are desirable experimental models for study of thermal and optical ET, in which single electron migration is ensured and the transferring electron is specified to be one of the $\delta$ electrons. ${ }^{27,28,29}$ Hereby, nine MV complexes of three series with a general formula $\left[\mathrm{Mo}_{2}(\mathrm{DAniF})_{3}\right]_{2}\left(\mu-4,4^{\prime}-\right.$ 
$\left.\mathrm{EE}^{\prime} \mathrm{C}\left(\mathrm{C}_{6} \mathrm{H}_{4}\right)_{n} \mathrm{CEE}^{\prime}\right)\left(\mathrm{DAniF}=N, N^{\prime}\right.$-di( $\left(p\right.$-anisyl)formamidinate, $\mathrm{E}, \mathrm{E}^{\prime}=\mathrm{O}$ or $\mathrm{S}$ and $\left.n=1-3\right)$, denoted as $\left[\mathbf{E E} \mathbf{E}^{\prime}-(\mathbf{p h})_{n}-\mathbf{E} \mathbf{E}^{\prime}\right]^{+}$(Fig. 1), were investigated as a testbed of the LZ theory. In Marcus theory, the total reorganization energy $\lambda$ is divided into $\lambda_{\text {in }}$ and $\lambda_{\text {out }}$, corresponding to the intramolecular $\left(\lambda_{\text {in }}\right)$ and solvent $\left(\lambda_{\text {out }}\right)$ nuclear motions..$^{5}\left[\mathbf{E E}-(\mathbf{p h})_{n}-\mathbf{E E}\right]^{+}$complexes have small $\lambda_{\text {in, }}$ as evidenced by the very low IVCT energy for [SS-ph-SS] ${ }^{+}\left(2640 \mathrm{~cm}^{-1}\right)^{27}$ in comparison with the Cruetz-Taube complex $\left(6369 \mathrm{~cm}^{-1}\right) .{ }^{18}$ Thus, the adiabaticity of the systems is effectively solvent-controlled because the $\lambda_{\text {in }}$ is generally assumed to be independent of the bridge length. ${ }^{30}$ This setup of the molecular systems permits to map the parameters $H_{\mathrm{ab}}$ and $\lambda$ throughout the adiabatic to the nonadiabatic limits. Incorporating the molecular and electronic dynamics of the thermal ET into the LZ model allows the nonadiabatic transition to be evaluated and the intermediate between the two limits to be characterized. Our results demonstrate that application of the LZ model in the adiabatic regime is not limited by $\Delta \mathrm{G}^{*}>>\left(H_{\mathrm{ab}}\right)$. Two intermediate systems, $\left[\mathbf{O S}-(\mathbf{p h})_{3}-\mathbf{O S}\right]^{+}$and $\left[\mathbf{S S}-(\mathbf{p h})_{3}-\mathbf{S S}\right]^{+}$, are identified with an overall transition probability of $\sim 0.5$ that is achieved through the single and the first multiple passage. This work has validated, for the first time, the LZ model and revealed the energetic and dynamic details of a system crossing over the two limits, which are not well described by this model. ${ }^{12,15}$ The results and conclusions unify the contemporary ET theories under the semiclassical framework. ${ }^{31}$

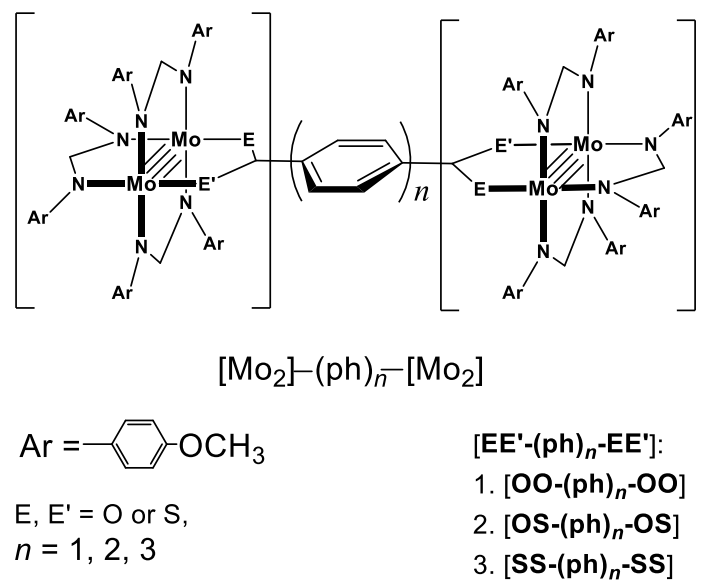

Fig. 1| A Molecular scaffold for the complexes under investigation. The three series of $\left[\mathrm{Mo}_{2}\right]-(\mathrm{ph})_{\mathrm{n}}$ $20\left[\mathrm{Mo}_{2}\right]$ complexes are differentiated by the $\left[\mathrm{Mo}_{2}\right]$ complex units due to $\mathrm{O} / \mathrm{S}$ alternation of the chelating atoms $\left(E\right.$ and $\left.E^{\prime}\right)$. Each series consists of three complexes with different (poly)phenylene bridges $\left(p h_{n}, n=1-3\right)$.

Using the published procedure for preparation of the $\mathrm{ph}$ and $\mathrm{ph}_{2}$ bridged analogues, ${ }^{27,28,32}$ three terphenylene-bridged $\mathrm{Mo}_{2}$ dimers in $\left[\mathbf{E} \mathbf{E}^{\prime}-(\mathbf{p h})_{3}-\mathbf{E E}\right]^{+}$were synthesized by assembling two 
$\mathrm{Mo}_{2}(\mathrm{DAniF})_{3}\left(\mathrm{O}_{2} \mathrm{CCH}_{3}\right)$ complexes with a bridging ligand, 4,4'-terphenyldicarboxylate or its thiolate derivatives, 4,4'-(EE'CC $\left.\mathrm{EE}_{18} \mathrm{H}_{12} \mathrm{CEE}^{\prime}\right)^{2-}\left(\mathrm{E} \mathrm{E}^{\prime}=\mathrm{O}\right.$ or S) (fig. S3-S4). The complexes were characterized by ${ }^{1} \mathrm{H}$ NMR spectroscopy (fig. S5-S7). The solid-state structure of [OS-(ph) - $\left._{3} \mathbf{- O S}\right]$ was determined by X-ray diffraction of a single-crystal. The X-ray crystal structure (fig. S8 and table $\mathrm{S} 1$ ) shows that the $\mathrm{O}$ and $\mathrm{S}$ chelating atoms are arranged in a trans manner, as in [OS-phOS]..$^{32}$ The average torsion angle between the neighboring ph groups is about $34^{\circ}$. The centroid distance between the two $\mathrm{Mo}_{2}$ complex units is $20.3 \AA$, and the edge to edge distance is $14.3 \AA$, as measured for the $\mathrm{C} \cdots \mathrm{C}$ distance between the two chelating groups. The $\mathrm{Mo}_{2} \cdots \mathrm{Mo}_{2}$ distances for [OO-(ph) $)_{3}-\mathbf{O O}$ ] [SS-(ph) 3-SS] $_{3}$ are estimated to be 19.74 and $20.74 \AA$, respectively, from the crystal structures of the associated complexes in series $\left[\mathbf{E E}^{\prime}-\mathbf{p h}-\mathbf{E} \mathbf{E}^{\prime}\right] .{ }^{32}$

The MV complexes $\left[\mathbf{E E}^{\prime}-(\mathbf{p h})_{\mathbf{n}}-\mathbf{E} \mathbf{E}^{\prime}\right]^{+}$were prepared by one-electron oxidation of the corresponding neutral compounds with one equivalent of ferrocenium hexafluorophosphate, ${ }^{27,28}$ which were analyzed in situ. These radical cations were characterized by X-band EPR spectra (fig. $\mathrm{S} 10)$, which exhibit one characteristic signal for ${ }^{96} \mathrm{Mo}(I=0)$ isotope with some weak hyperfine structures from ${ }^{95} \mathrm{Mo}(I=5 / 2)$ and ${ }^{97} \mathrm{Mo}(I=5 / 2)$. The EPR peaks center at $\mathrm{g}=1.951$ ([OO-(ph) $)^{-}$ $\left.\mathbf{O O}]^{+}\right), 1.953\left(\left[\mathbf{O S}-(\mathbf{p h})_{3}-\mathbf{O S}\right]^{+}\right)$and $1.956\left(\left[\mathbf{S S}-(\mathbf{p h})_{3}-\mathbf{S S}\right]^{+}\right)$, smaller than that for an organic radical, indicating that the odd electron resides essentially on a $\delta$ orbital. ${ }^{26}$ The g values increase as the chelating atoms $\mathrm{O}$ are replaced by $\mathrm{S}$ atoms, as seen for the $\mathrm{ph}^{27}$ and $\mathrm{ph}_{2}{ }^{28}$ series. It is noted that the $\mathrm{g}$ values for the $\mathrm{ph}_{3}$ bridged complexes are appreciably large, while smaller $\mathrm{g}$ values are obtained for the $\mathrm{ph}$ and $\mathrm{ph}_{2}$ series. For these localized MV complexes one would expect smaller $\mathrm{g}$ values. Increase of the $\mathrm{g}$ values implies that the odd electron spends more time on the $\mathrm{ph}_{3}$ bridge.

For the $\mathrm{Mo}_{2}$ dimers, the charge transfer spectra from visible to IR region are pertinent to the $\delta$ electron transition. The MV complexes $\left[\mathbf{E E}^{\prime}-(\mathbf{p h}) \mathbf{n}-\mathbf{E E}^{\prime}\right]^{+}$exhibit a metal $(\delta)$ to bridging ligand $\left(\pi^{*}\right)$ charge transfer (MLCT) absorption in the visible region as the neutral precursors with essentially the same transition energy $\left(E_{\mathrm{ML}}\right)$ but substantially reduced band intensity. ${ }^{27,28,32}$ The MLCT band is red shifted with increasing S chelating atoms and blue shifted as the bridge is lengthened (fig. S11, Table 1). ${ }^{27,28,32}$ For $\left[\mathbf{E E}^{\prime}-(\mathbf{p h}) \mathbf{n}-\mathbf{E} \mathbf{E}^{\prime}\right]^{+}$with the same ancillary DAniF ligands, the vertical $\delta \rightarrow \delta^{*}$ transition occurs at similar wavenumber, ca. $\sim 450 \mathrm{~nm} ;{ }^{26}$ however, this band is masked sometimes by the other electronic transitions. ${ }^{27}$ For example, careful examination of the spectrum of [OO-(ph) $\left.)_{3}-\mathbf{O O}\right]$ founds that the absorbance in the $400-600 \mathrm{~nm}$ region results from an overlap of the $\delta \rightarrow \delta^{*}$ transition at $446 \mathrm{~nm}\left(\varepsilon=750 \mathrm{M}^{-1} \mathrm{~cm}^{-1}\right)$ and the MLCT at $450 \mathrm{~nm}\left(\varepsilon_{\mathrm{ML}}\right.$ 
$=450 \mathrm{M}^{-1} \mathrm{~cm}^{-1}$ ) (fig. S11A). For $[\mathbf{O S}-\mathbf{p h}-\mathbf{O S}]^{+}$and $[\text {SS-ph-SS }]^{+}$, a ligand to metal charge transfer (LMCT) absorption was observed with the transition energy lower than that of the MLCT band. ${ }^{27}$ The LMCT band for the MV complexes arises from charge transfer from the $\pi$ orbital of bridging ligand to the $\delta$ orbital of the cationic $\mathrm{Mo}_{2}$ center, thus, corresponding to hole transfer in the opposite direction. Simultaneous presence of the MLCT and LMCT bands facilitates the throughbond superexchange, ${ }^{33}$ leading to strong EC between the two $\mathrm{Mo}_{2}$ centers. ${ }^{32}$

The MV dimers with ph and $\mathrm{ph}_{2}$ bridges present a characteristic IVCT band, from which the spectral parameters, transition energy $\left(E_{\mathrm{IT}}\right)$, molar extinction coefficient $\left(\varepsilon_{\mathrm{IT}}\right)$ and half-high

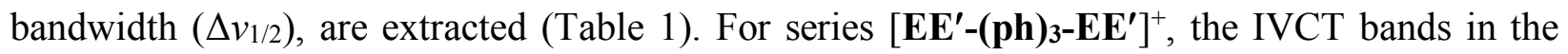
near-IR region are extremely weak, particularly for $\left[\mathbf{O O}-(\mathbf{p h})_{3}-\mathbf{O O O}\right]^{+}$(fig. S11). The general variation trends of the IVCT bands for these series are: red shifting of the absorption with increase of S chelating atoms and blue shifting with elongating the bridge (Table 1), showing the two factors that affect the electronic coupling. ${ }^{27,28}$ Compared to those with the same $\left[\mathrm{Mo}_{2}\right]$ units, series $\left[\mathbf{E E}^{\prime}-\right.$ (ph) $\left.)_{3}-\mathbf{E E}\right]^{+}$exhibits the IVCT bands with the highest transition energies, that is, $E_{\mathrm{IT}}=12400 \mathrm{~cm}^{-1}$ $\left(\left[\mathbf{O O}-(\mathbf{p h})_{3}-\mathbf{O O}\right]^{+}\right), 7400 \mathrm{~cm}^{-1}\left(\left[\mathbf{O S}-(\mathbf{p h})_{3}-\mathbf{O S}\right]^{+}\right)$and $6200 \mathrm{~cm}^{-1}\left(\left[\mathbf{S S}-(\mathbf{p h})_{3}-\mathbf{S S}\right]^{+}\right)($Table 1). The more delocalized $\left[\mathbf{S S}-(\mathbf{p h})_{3}-\mathbf{S S}\right]^{+}$has a $E_{\text {IT }}$ comparable to that for the organic MV D-B-A system with a $\mathrm{ph}_{3}$ bridge $\left(6700 \mathrm{~cm}^{-1}\right){ }^{34}$ It is interesting that the IVCT bands for $\left[\mathbf{E E}^{\prime}-(\mathbf{p h})_{3}-\mathbf{E E}^{\prime}\right]^{+}$are narrower than those of the $\mathrm{ph}_{2}$ analogues (Table 1). This is phenomenal because IVCT band broadening is expected for weaker coupling systems according to $\Delta \nu^{0}{ }_{1 / 2}=2[4 \ln (2) \lambda R T]^{1 / 2} \cdot 9,35$

The EC constants $\left(H_{\mathrm{ab}}\right)$ are calculated from the Mulliken-Hush expression (eq. 2). ${ }^{9,19}$ In application of eq. 2 for $\left[\mathbf{E} \mathbf{E}^{\prime}-(\mathbf{p h})_{n}-\mathbf{E} \mathbf{E}^{\prime}\right]^{+}$, the length of the bridge has been used as the effective ET distance, considering that the $\delta$ electrons are fully delocalized over the $\left[\mathrm{Mo}_{2}\right]$ coordination shell. Therefore, for the $\mathrm{ph}, \mathrm{ph}_{2}$ and $\mathrm{ph}_{3}$ series, the geometrical lengths of the bridge " $-\left(\mathrm{C}_{6} \mathrm{H}_{4}\right)_{n}{ }^{-}$", 5.8, 10.0 and $14.3 \AA$, respectively, are adopted to be $r_{\mathrm{ab}}$ for the given systems. ${ }^{27,28}$ The $H_{\mathrm{ab}}$ data are listed in Table 1. Large decrease of $H_{\mathrm{ab}}$ is found for the $\left[\mathbf{E} \mathbf{E}^{\prime}-(\mathbf{p h})_{3}-\mathbf{E} \mathbf{E}^{\prime}\right]^{+}$complexes (Table 1). $H_{\mathrm{ab}}=120 \mathrm{~cm}^{-1}$ and $134 \mathrm{~cm}^{-1}$ are determined for $\left[\mathbf{O S}-(\mathbf{p h})_{3}-\mathbf{O S}\right]^{+}$and $\left[\mathbf{S S}-(\mathbf{p h})_{3}-\mathbf{S S}\right]^{+}$, respectively. For $\left[\mathbf{O O}-(\mathbf{p h})_{3}-\mathbf{O O}\right]^{+}$, the $H_{\mathrm{ab}}$ of $62 \mathrm{~cm}^{-1}$ is confirmed by calculate result from the CNS formula $\left(63 \mathrm{~cm}^{-1}\right)$ (fig. S19), ${ }^{23,36}$ the alternative approach developed by Creutz, Newton, and Sutin. It is worthwhile to note that similar $H_{\mathrm{ab}}$ values are obtained for $\left[\mathbf{O S}-(\mathbf{p h})_{3}-\mathbf{O S}\right]^{+}$and $\left[\mathbf{S S}-(\mathbf{p h})_{3}-\mathbf{S S}\right]^{+}$, while the magnitudes of $E_{\mathrm{IT}}$ are substantially different, implying that the matrix elements are 
independent of the nuclear geometries. This phenomenon conforms to the Condon approximation, manifesting a system transition from adiabatic to nonadiabatic, but contradicts the theoretical outcomes with calculated matrix elements. ${ }^{37}$ Optical analysis indicates that these two systems belong to the weak coupling Class II, while $\left[\mathbf{O O}-(\mathbf{p h})_{3}-\mathbf{O O}\right]^{+}$should be assigned to Class I, in terms of the Robin-Day's scheme. ${ }^{9,38}$ 
Table 1. Spectroscopic and ET kinetic data ${ }^{a}$ and the LZ parameters ${ }^{b}$ for mixed-valence complexes $\left[\mathbf{E E}^{\prime}-(\mathbf{p h})_{\mathbf{n}}-\mathbf{E E}^{\prime}\right]^{+}\left(\mathrm{E} \mathrm{E} \mathrm{E}^{\prime}=\mathrm{O}\right.$ or $\mathrm{S}$ and $n=1-3){ }^{c}$

\begin{tabular}{|c|c|c|c|c|c|c|c|c|c|}
\hline & \multicolumn{3}{|c|}{$\left[\mathbf{E E} \mathbf{E}^{\prime}-\mathbf{p h}-\mathbf{E} \mathbf{E}^{\prime}\right]^{+}$} & \multicolumn{3}{|c|}{$\left[\mathbf{E E}^{\prime}-(\mathbf{p h})_{2}-\mathbf{E E}^{\prime}\right]^{+}$} & \multicolumn{3}{|c|}{$\left[\mathbf{E E ^ { \prime }}-(\mathbf{p h})_{3}-\mathbf{E} \mathbf{E}^{\prime}\right]^{+}$} \\
\hline & {$[\mathbf{O O}]$} & [OS] & {$[\mathbf{S S}]$} & {$[\mathbf{O O}]$} & [OS] & [SS] & {$[\mathbf{O O}]^{d}$} & [OS] & [SS] \\
\hline$E_{\mathrm{IT}}\left(\mathrm{cm}^{-1}\right)$ & 4240 & 3440 & 2640 & 8300 & 6536 & 4826 & 12400 & 7400 & 6200 \\
\hline$\varepsilon_{\mathrm{IT}}\left(\mathrm{M}^{-1} \mathrm{~cm}^{-1}\right)$ & 1470 & 3690 & 12660 & 198 & 715 & 1610 & 50 & 220 & 315 \\
\hline$\Delta v_{1 / 2}\left(\mathrm{~cm}^{-1}\right)$ & 4410 & 3290 & 1770 & 5185 & 6338 & 5231 & 3010 & 4210 & 4421 \\
\hline$\lambda / 4\left(\mathrm{~cm}^{-1}\right)$ & 1060 & 860 & 660 & 2075 & 1634 & 1206 & 3100 & 1850 & 1550 \\
\hline$\Delta G^{*}\left(\mathrm{~cm}^{-1}\right)$ & 581 & 266 & 79 & 1889 & 1299 & 827 & 3038 & 1732 & 1422 \\
\hline$\left(\lambda / 4-H_{\mathrm{ab}}\right)$ & 471 & 134 & -204 & 1885 & 1288 & 791 & 3038 & 1731 & 1416 \\
\hline$k_{\mathrm{et}}(\mathrm{ad})\left(\mathrm{s}^{-1}\right)$ & $3.0 \times 10^{11}$ & $1.4 \times 10^{12}$ & $3.4 \times 10^{12}$ & $4.1 \times 10^{8}$ & $9.3 \times 10^{9}$ & $9.2 \times 10^{10}$ & $3.0 \times 10^{5}$ & $5.6 \times 10^{8}$ & $3.0 \times 10^{9}$ \\
\hline$P_{0}$ & 1 & 1 & 1 & 0.60 & 0.97 & 1 & 0.076 & 0.32 & 0.41 \\
\hline$\kappa_{\mathrm{el}}$ & 1 & 1 & 1 & 0.75 & 0.98 & 1 & 0.14 & 0.48 & 0.58 \\
\hline
\end{tabular}

${ }^{a}$ For $\left[\mathbf{E E}^{\prime}-\mathbf{p h}-\mathbf{E} \mathbf{E}^{\prime}\right]^{+}$and $\left[\mathbf{E E}-(\mathbf{p h})_{\mathbf{2}}-\mathbf{E} \mathbf{E}^{\prime}\right]^{+}$, the spectroscopic and ET kinetic Data are cited from ref.27,28. Data extraction and derivation are shown in fig. S12-17. ${ }^{b}$ The LZ parameters are calculated from eq $1 .{ }^{c}$ For all the calculations, an average nuclear vibrational frequency, $v_{\mathrm{n}}=5 \times 10^{12} \mathrm{~s}^{-1}$, is adopted (ref. 9). ${ }^{d} r_{\mathrm{c}-\mathrm{c}}$ refers to the center to center separation between the two Mo 2 centers.

${ }^{e}$ Electronic transition frequencies $\left(v_{\mathrm{el}}\right)$ are calculated by eq. 3 
The MV [ $\left.\mathrm{Mo}_{2}\right]$-bridge-[ $\left.\mathrm{Mo}_{2}\right]$ complex constitutes uniquely an effective "one-particle" donoracceptor system. ${ }^{13}$ In such as a system, adopting a semiclassical two-state LZ model, ${ }^{10,11}$ the ET initial $\left(\phi_{\mathrm{I}}\right)$ and final $\left(\phi_{\mathrm{F}}\right)$ diabatic states can be approximated by the $\delta$ orbtials of the donor and acceptor, namely, $\delta_{\mathrm{D}}$ and $\delta_{\mathrm{A}}$, respectively. Assuming that the diabatic and adiabatic states essentially coincide in the vicinity of the electronic equilibrium configurations, linear combinations of $\delta_{\mathrm{D}}$ and $\delta_{\mathrm{A}}$ generate two first-order or adiabatic states (eq 4$),{ }^{29}$

$$
\begin{aligned}
& \Psi_{1}=c_{\mathrm{a}} \delta_{\mathrm{D}}+c_{\mathrm{b}} \delta_{\mathrm{A}}=(1 / 2)^{1 / 2}\left(\delta_{\mathrm{D}}+\delta_{\mathrm{A}}\right) \\
& \Psi_{2}=c_{\mathrm{a}} \delta_{\mathrm{D}}-c_{\mathrm{b}} \delta_{\mathrm{A}}=(1 / 2)^{1 / 2}\left(\delta_{\mathrm{D}}-\delta_{\mathrm{A}}\right)
\end{aligned}
$$

Then, we have the nonadiabatic mixing matrix element

$$
H_{\mathrm{ab}}=\left\langle\delta_{\mathrm{D}}|h| \delta_{\mathrm{A}}\right\rangle
$$

where $h$ is an effective one-electron Hamiltonian. ${ }^{13,29}$ The energies of the adiabatic states, obtained by solving the two-state secular determinant, are given by eq $5,{ }^{24,35}$

$$
\begin{aligned}
& V_{1}=\frac{\left[\lambda\left(2 X^{2}-2 X+1\right)+\Delta G^{0}\right]}{2}+\frac{\left[\left(\lambda(2 X-1)-\Delta G^{0}\right)^{2}+4 H_{D A}^{2}\right]^{1 / 2}}{2} \\
& V_{2}=\frac{\left[\lambda\left(2 X^{2}-2 X+1\right)+\Delta G^{o}\right]}{2}-\frac{\left[\left(\lambda(2 X-1)-\Delta G^{o}\right)^{2}+4 H_{D A}^{2}\right]^{1 / 2}}{2}
\end{aligned}
$$

where $\Delta \mathrm{G}^{\circ}=0$ for the current symmetrical systems. These two adiabatic states are represented by the upper $\left(V_{1}\right)$ and lower $\left(V_{2}\right)$ PESs separated by $2 H_{\mathrm{ab}}$ at $\mathrm{X}=0.5 .^{9,13,35}$ Study of the strongly coupled systems $\left[\mathbf{E E}^{\prime}-\mathbf{E} \mathbf{E}^{\prime}\right]^{+}(n=0)$ has demonstrated that the upper and lower curves of the adiabatic potential diagram evolve into the electronic energy levels HOMO $(\delta+\delta)$ and HOMO-1 $(\delta-\delta) .{ }^{29}$ In this strongly coupled limit, [SS-SS $]^{+}$, the HOMO-HOMO-1 gap $\left(\Delta E_{\mathrm{H}-\mathrm{H}-1}\right)$ equals exactly the 20 measured "IVCT" energy in the spectra and the $2 H_{\mathrm{ab}}$ calculated from the modified Mulliken-Hush expression for Class III system, ${ }^{9,13,35,39}$ which justify the $\delta$ orbitals as the basis of the zero-order wavefunctions of the initial and final diabatic states for the thermal ET for the $\mathrm{Mo}_{2} \mathrm{D}-\mathrm{B}-\mathrm{A}$ system.

Analysis of the vibronic bands gives rise to the $\lambda$ and $H_{\mathrm{ab}}$ for constructions of the adiabatic PESs from eq 5. Shown in Fig. 2 are the adiabatic PES diagrams for three series, [OO- $(\mathbf{p h})_{n-}$ $25 \mathbf{O O}^{+}$(Fig. 2A), [SS-(ph) $\left.{ }_{n}-\mathbf{S S}\right]^{+}$(Fig. 2B) and $\left[\mathbf{E E}^{\prime}-(\mathbf{p h})_{2}-\mathbf{E E}^{\prime}\right]^{+}$(Fig. 2C). These reaction potential diagrams interpret well the IVCT band characteristics. As shown in Fig. 2A, The three systems in $\left[\mathbf{O O}-(\mathbf{p h})_{n}-\mathbf{O O}\right]^{+}$present double-well PESs differentiated by the vibronic transition energy $\left(E_{\mathrm{IT}}\right)$ and the adiabatic splitting $2 H_{\mathrm{ab}} \cdot\left[\mathbf{O O}-(\mathbf{p h})_{3}-\mathbf{O O}\right]^{+}$, as the most weakly coupled 
system, features small curvatures of the diabatic parabolic potential curves. The adiabatic PESs coincide with the diabatic PESs in the conical region with the upper $\left(V_{1}\right)$ and lower $\left(V_{2}\right)$ surfaces meeting almost at the diabatic crossing point (Fig. 2A). In series $\left[\mathbf{S S}-(\mathbf{p h})_{n} \text {-SS }\right]^{+}$(Fig. 2B), the PESs for [SS-ph-SS $]^{+}$are dramatically different from those of the systems with longer bridges. It shows nearly a flat lower $V_{2}$ surface with two very shallow wells at the reactant and product equilibriums. The separation between $V_{1}$ and $V_{2}$ at $\mathrm{X}=0$ corresponds to the low Frank-Condon transition energy $\left(E_{\mathrm{IT}}=\lambda\right)$, close to the adiabatic spacing $\left(2 H_{\mathrm{ab}}\right)$ at $\mathrm{X}=0.5$ (Table 1$)$. The transition state energy $\left(\Delta \mathrm{G}^{*}\right)$ is only $79 \mathrm{~cm}^{-1}$, much less than the thermal energy level $k_{\mathrm{B}} T\left(207 \mathrm{~cm}^{-1}\right.$ at 298 $K$ ). This causes the thermal energy level unevenly populated around the reactant equillibrium; consequently, Frank-Condon transition generates a "half cutting-off" IVCT band (Table 1) 27,32 typically for Class II-III transitional MV systems. ${ }^{24,32,40}$ For $\left[\mathbf{S S}-(\mathbf{p h})_{3}-\mathbf{S S}\right]^{+}$, on the other hand, the outspreading shift of the reactant and product equilibriums and the small curvature of the energy parabola account for the high-energy, narrowed IVCT band, signaling the turning point of system from the solvent-controlled adiabatic to the nonadiabatic regime. Series $\left[\mathbf{E E} \mathbf{E}^{\prime}-(\mathbf{p h})_{2}-\mathbf{E E}^{\prime}\right]^{+}$(Fig. $2 \mathrm{C}$ ), with the same $\mathrm{ph}_{2}$ bridge, shows that the $\mathrm{S}$ chelating atoms enhance effectively the EC by lowering $\lambda$ and increasing $2 H_{\mathrm{ab}}$.
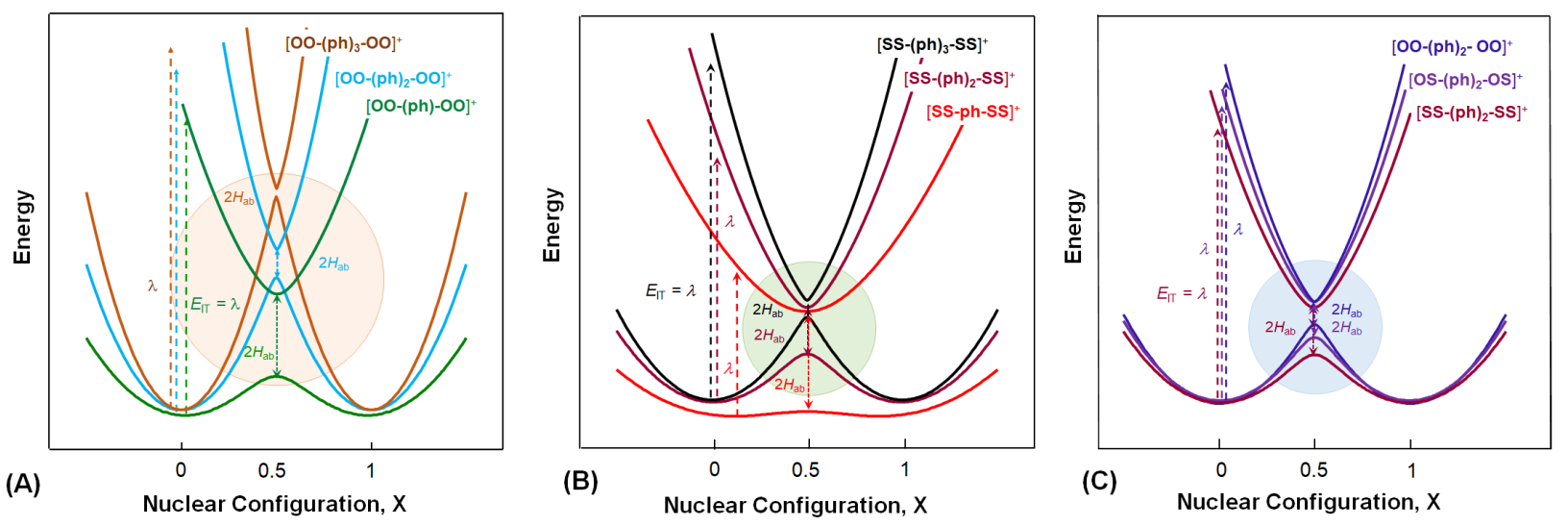

Fig. 2| Diagrams of the adiabatic potential energy surfaces for the mixed-valence $\left\{\left[\mathrm{Mo}_{2}\right]-(\mathrm{ph})_{n^{-}}\right.$

[Mo $\left._{2}\right\}^{+}$derived from eq 5 based on the optically determined $\lambda$ and $\boldsymbol{H}_{\mathrm{ab}}$ (A), Series [OO-(ph) $\left.n-0 O\right]^{+}(n$ $=1-3) .(B)$, Series $\left[S S-(p h)_{n}-S S\right]^{+}(n=1-3) .(C)$, Series $\left[E E^{\prime}-(p h)_{2}-E E^{\prime}\right]^{+}\left(E, E^{\prime}=0\right.$ or S). For each of the PES diagrams, the avoided crossing area is highlighted. 
According to Marcus, ${ }^{3,5}$ in the nonadiabatic limit, the thermal activation energy $\Delta G^{*}=(\lambda+$ $\left.\Delta G^{\circ}\right)^{2} / 4 \lambda$; in the adiabatic limit, $\Delta G^{*}$ is reduced by $H_{\text {ab. }}{ }^{9,13}$ Since $\left(\lambda+\Delta G^{\circ}\right)^{2} / 4 \lambda$ in the nonadiabatic limit is a value of the lowest (i.e., zeroth) order in $H_{\mathrm{ab}}$, we can reasonably approximate $\Delta G^{*}$ by eq 7 for the adiabatic-nonadiabatic borderline regime when $H_{\mathrm{ab}}$ is sufficiently small. ${ }^{2}$

$$
\Delta G^{*}=\left(\lambda+\Delta G^{\circ}\right)^{2} / 4 \lambda-H_{\mathrm{ab}}
$$

For symmetrical system, we have

$$
\Delta G^{*}=\lambda / 4-H_{\mathrm{ab}}
$$

Then, the difference between $\lambda / 4$ and $\Delta G^{*}$, i.e., $\left(\lambda / 4-\Delta G^{*}\right)$, is expected to equal $H_{\mathrm{ab}}$,

$$
H_{\mathrm{ab}}=\lambda / 4-\Delta G^{*}
$$

These energetic relationships (eqs 7 and 8) show the important correlation between $\Delta G^{\circ}, \Delta G^{*}, \lambda$ and $H_{\mathrm{ab}}$, for the transient system and thus, can be used as a quantitative probe of the crossover intermediate. Table 1 lists the values of $\left(\lambda / 4-H_{\mathrm{ab}}\right)$ for each of the systems, in comparison with $\Delta G^{*}$. Obviously, such a correlation does not exist for strongly coupled systems, for example, [EE'ph-EE' $]^{+}$(Table 1). For each series, the deviation between $\Delta G^{*}$ and $\left(\lambda / 4-H_{\mathrm{ab}}\right)$ decreases as the system nonadiabaticity increases with elongating the bridge. Remarkably, the $\left(\lambda / 4-H_{\mathrm{ab}}\right)$ values, for $\left[\mathbf{O O}-(\mathbf{p h})_{2}-\mathbf{O O}\right]^{+},\left[\mathbf{O S}-(\mathbf{p h})_{3}-\mathbf{O S}\right]^{+}$and $\left[\mathbf{S S}-(\mathbf{p h})_{3}-\mathbf{S S}\right]^{+}$, are essentially equal to the $\Delta G^{*}$ s. For the most weakly coupled $\left[\mathbf{O O}-(\mathbf{p h})_{3}-\mathbf{O O}\right]^{+}, \Delta G^{*}$ and $\left(\lambda / 4-H_{\mathrm{ab}}\right)$ have exactly the same value, $3071 \mathrm{~cm}^{-1} ;\left(\lambda / 4-\Delta G^{*}\right)=62 \mathrm{~cm}^{-1}$, precisely equaling the $H_{\mathrm{ab}}$ (Table 1$)$. These results represent the energetic features of systems in transition from the adiabatic to nonadiabatic limit.

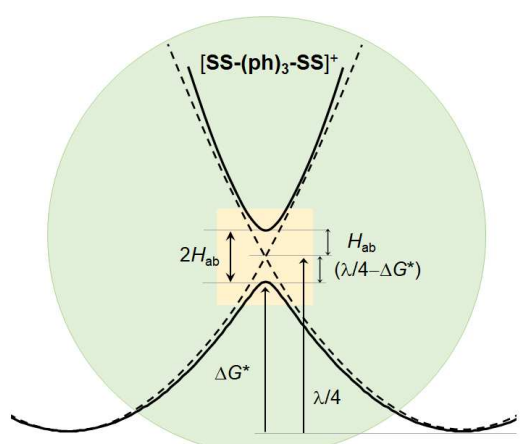

(A)

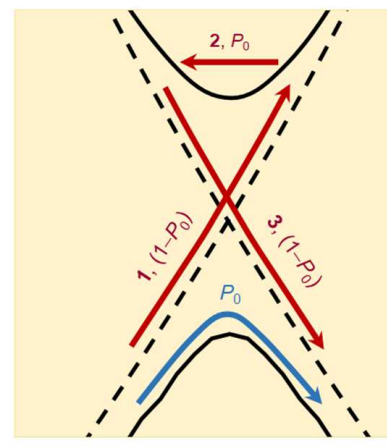

(B)

Fig. 3| Schematic representations of the energetic features of the crossing region and nonadiabatic transition channels for the transient systems. (A), The diabatic (dashed line) and adiabatic (solid line) potential energy surfaces in the reactant and product equilibriums and the transition state for [SS-(ph)3SS] ${ }^{+}$. (B), The first (cyan) and second (red) channels of nonadiabatic transition in intermediate system [SS- 
$\left.(\mathrm{ph})_{3}-\mathrm{SS}\right]^{+}$. The potential surfaces in $(\mathrm{A})$ and $(\mathrm{B})$ result from zooming in of the avoided crossing area in Fig. 2B. Similar results are expected for [OS-(ph) $\left.{ }_{3}-\mathbf{O S}\right]^{+}$from the similar $\lambda$ and $H_{\mathrm{ab}}$ data.

The adiabatic ET rate constants, $k_{\mathrm{et}}(\mathrm{ad})$, for the MV systems are calculated from the classical transition state formalism ${ }^{1,5,9}$ (eq. 9) with a preexponential factor $\kappa_{\mathrm{el}} \nu_{\mathrm{n}}$ and activation energy $\left(\Delta \mathrm{G}^{*}\right)$ from the Marcus-Hush theory (eq. 10). $.9,39$

$$
\begin{aligned}
& k_{\mathrm{et}}=\kappa_{\mathrm{el}} v_{\mathrm{n}} \exp \left(-\frac{\Delta G^{*}}{k_{\mathrm{B}} T}\right) \\
& \Delta G^{*}=\frac{\left(\lambda-2 H_{\mathrm{ab}}\right)^{2}}{4 \lambda}
\end{aligned}
$$

The nonadiabatic ET rate constants, $k_{\text {et }}$ (nonad), can be determined by the Levich-Marcus expression (eq. 11): $:$,5,21,41

$$
k_{\mathrm{et}}=\frac{2 H_{\mathrm{ab}}^{2}}{h} \sqrt{\frac{\pi^{3}}{\lambda k_{\mathrm{B}} T}} \exp \left(-\frac{\lambda}{4 k_{\mathrm{B}} T}\right)
$$

The accuracy of the optically determined rate constants is confirmed by IR-band broadening analysis recently. ${ }^{42}$ In this work, a transmission coefficient $\left(\kappa_{\mathrm{el}}=1-0.11\right)$ calculated from the LZ formula (eq. 1) (Table 1) is used to derive $k_{\mathrm{et}}(\mathrm{ad})$ from eq 9. Given the low-frequency solvent modes $v_{\text {out }}$ in $10^{12}-10^{13} \mathrm{~s}^{-1}$ in classical theory, an averaged nuclear frequency, $v_{\mathrm{n}}=5 \times 10^{12} \mathrm{~s}^{-1}$ is generally adopted. ${ }^{9,23}$ This is further justified in the present systems in which the nonadiabatic transition is governed by solvent thermal fluctuations. In the nonadiabatic limit, comparison of eq 11 to eq 9 , in conjunction with eq 1 a, gives $\kappa=2(2 \pi \gamma)$ and $\Delta G^{*}=\lambda / 4$, the Marcus activation erengy. ${ }^{5,13}$ This indicates implicitly that the adiabatic and nonadiabatic limits are bridged through the intermediate of the LZ model, which can be exploited to test the connection of the existing ET rate expressions in the two limits.

For the ph and $(\mathrm{ph})_{2}$ bridged series (Table 1), the electron frequencies $\left(v_{\mathrm{el}}\right)$ are in the order of $10^{13}-10^{14} \mathrm{~s}^{-1}$, higher than the nuclear vibrational frequency $\left(v_{\mathrm{n}}\right)\left(10^{12}-10^{13} \mathrm{~s}^{-1}\right)$ by one order of magnitude. [SS-ph-SS $]^{+}$has the highest ET rate with $k_{\mathrm{et}}(\mathrm{ad})=3.4 \times 10^{12} \mathrm{~s}^{-1}$, close to the adiabatic limit $\left(5 \times 10^{12} \mathrm{~s}^{-1}\right)$, in accordance with its optical behavior as a Class II-III MV system. However, the rate constant derived from eq $11, k_{\text {et }}($ nonad $)=1.4 \times 10^{14} \mathrm{~s}^{-1}$, is significantly larger than $v_{\mathrm{n}}$, indicating the irrationality of the nonadiabatic treatment for this system (Table 1). The deviation of $k_{\mathrm{et}}(\mathrm{nonad})$ from $k_{\mathrm{et}}(\mathrm{ad})$ decreases with increase of the nonadiabaticity. It is remarkable that for 
the transient systems, $\left[\mathbf{O O}-(\mathbf{p h})_{2}-\mathbf{O O}\right]^{+}$and $\left[\mathbf{E} \mathbf{E}^{\prime}-(\mathbf{p h})_{3}-\mathbf{E} \mathbf{E}^{\prime}\right]^{+}, k_{\mathrm{et}}(\mathrm{ad})=k_{\mathrm{et}}($ nonad) with small analytical errors, and the data fall in the range of $10^{8}-10^{9} \mathrm{~s}^{-1}$ (Table 1). Similarly, $k_{\mathrm{et}} \sim 10^{9} \mathrm{~s}^{-1}$ is reported for the $\mathrm{ph}_{3}$ bridged organic radical system. ${ }^{34}$ It is noted that the rate constant for [SS(ph) $\left.)_{3} \mathbf{- S S}\right]^{+}$is about 5 times larger than that of $\left[\mathbf{O S}-(\mathbf{p h})_{3}-\mathbf{O S}\right]^{+}$(Table 1), despite the similar $H_{\mathrm{ab}}$ values. The high sensitivity of $k_{\mathrm{et}}$ on $H_{\mathrm{ab}}$ is consistent with the increased nonadiabaticity for these systems. ${ }^{1,2,5,15,30}$ In contrast, the strongly coupled series $\left[\mathbf{E E}-\mathbf{- p h}-\mathbf{E} \mathbf{E}^{\prime}\right]^{+}$shows $H_{\mathrm{ab}}$ independence of the $k_{\text {et, }}$ (Table 1), as expected. ${ }^{2,10,11,15,30}$ Importantly, the kinetic data demonstrate that the adiabatic and nonadiabatic regimes are smoothly bridged by the crossover regime, which can be well described by the Marcus theory. ${ }^{5,24}$ The nonadiabatic treatments using solely the average lowfrequency nuclear mode $\left(v_{n}\right)$ on the thermal ET occurring at the intersection of the adiabatic PESs generate precisely consistent outcomes in both the nondiabetic limit and the transient regime. Therefore, this work shows that the adiabatic and nonadiabatic ET rate expressions are applicable in the respective ET dynamic limits, and work equally well with accordant results for the LZ intermediates, although a single theory that rigorously treats the two limits is not available. ${ }^{16,31}$
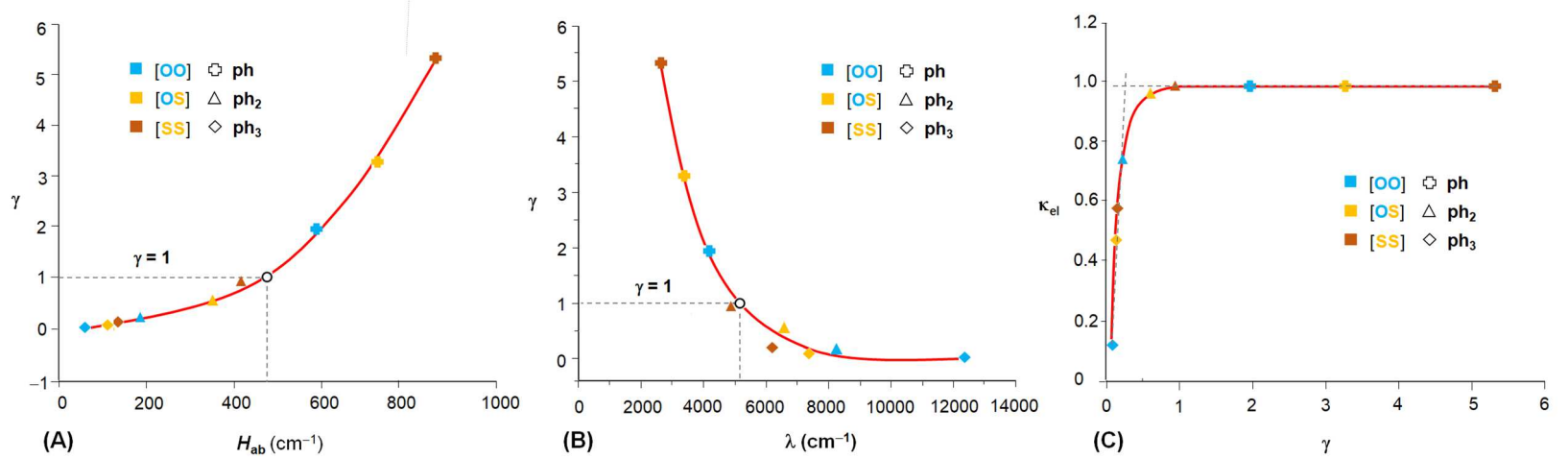

Fig. 4| Impacts of the electronic and nuclear factors on the Landau-Zener parameters $\gamma$ and $\kappa_{e l}$ in systems $\left[E E^{\prime}-(p h)_{n}-E E^{\prime}\right]^{+}\left(E, E^{\prime}=0\right.$ or $\left.S, n=1-3\right)$. (A). Variation of the adiabatic parameter $\gamma$ as a function of the transfer integral $\left(H_{\mathrm{ab}}\right)$. (B) Variation of the transmission coefficient $\kappa_{\mathrm{el}}$ as a function of reorganization energy ( $\lambda$ ). (C). $\gamma$-dependence of Kel. Color codes for [EE'-(ph) $\left.n^{-} E^{\prime}\right]^{+}$: blue for [OO-(ph) $\left.n-00\right]^{+}$, yellow for [OS-(ph) $\left.{ }_{n}-\mathbf{O S}\right]^{+}$and brown for $\left[\mathbf{S S}-(\mathbf{p h})_{n}-\mathbf{S S}\right]^{+}$. The $\mathrm{ph}, \mathrm{ph}_{2}$ and $\mathrm{ph}_{3}$ bridges are represented by cross, triangle and square, respectively.

The impacts of $H_{\mathrm{ab}}$ and $\lambda$ on $\gamma$ and $\kappa_{\mathrm{el}}$ are schematically presented in Fig. 4, which show the smooth systematic transformation from the adiabatic to the nonadiabatic limit. Complexes in [EE'ph-EE' $]^{+}$are in the adiabatic limit with $\kappa_{\mathrm{el}}=1$ and $\gamma=2-5(>>1)$ due to the short bridge. In series $\left[\mathbf{E E}^{\prime}-(\mathbf{p h})_{2}-\mathbf{E E}\right]^{+},\left[\mathbf{S S}-(\mathbf{p h})_{2}-\mathbf{S S}\right]^{+}$has a unity transmission coefficient but the $\gamma$ is lowered to 0.91, 
while for $\left[\mathbf{O O}-(\mathbf{p h})_{2}-\mathbf{O O}\right]^{+}$, both $\kappa_{\mathrm{el}}$ and $\gamma$ are significantly lower than 1 . With $\gamma=0.013(<<1)$, $\left[\mathbf{O O}-(\mathbf{p h})_{3}-\mathbf{O O}\right]^{+}$should be placed in the nonadiabatic regime. This is confirmed by the $0.14 \kappa_{\mathrm{el}}$ value, which is close to the nonadiabatic pre-exponential factor $(0.16)$ calculated from $\kappa=4 \pi \gamma \cdot{ }^{1,2}$ While $\gamma>1$ and $\gamma<<1$ characterize the adiabatic and nonadiabatic limits, respectively, the intermediate is not explicitly classified in the LZ model. For $\left[\mathbf{O S}-(\mathbf{p h})_{3}-\mathbf{O S}\right]^{+}(\gamma=0.065)$ and $[\mathbf{S S}-$ (ph) $\left.{ }_{3}-\mathbf{S S}\right]^{+}(\gamma=0.084), \gamma$ is much less than 1 , from which the systems might be assigned to the nonadiabatic limit. However, for both, $\kappa_{\mathrm{el}} \approx 0.5$, meaning that about $50 \%$ of the transition attempts that reach the transition state through thermal fluctuation can successfully complete the ET process. We have seen that these systems present dynamic and energetic properties in the avoided area that are distinct from those in the adiabatic and nonadiabatic limits. Fig. 4 shows clearly the transient status of the systems. Therefore, $\kappa_{\mathrm{el}} \approx 0.5$ can be considered to be the practical criterion to identify the transient system, whilst the LZ model ${ }^{10,11}$ and other theories ${ }^{14,15,16}$ do not provide such a solution on this issue. Newton and Sutin pointed out that when $H_{\mathrm{ab}}>200 \mathrm{~cm}^{-1}, \kappa_{\mathrm{el}} \geq 0.6$ for typical transition metal redox systems. ${ }^{1}$ Here, for these two systems with $H_{\mathrm{ab}}=120$ and 130 $15 \mathrm{~cm}^{-1}$, the $\mathrm{K}_{\mathrm{el}}$ values 0.5 and 0.6 are in excellent agreement with the theoretic predications. For the three $\left[\mathbf{E E}^{\prime}-(\mathbf{p h})_{3}-\mathbf{E E}\right]^{+}$complexes and $\left[\mathbf{O O}-(\mathbf{p h})_{2}-\mathbf{O O}\right]^{+}$, a liner relationship between $\kappa_{\mathrm{el}}$ and $\gamma$ is found (Fig. 4C), for which $\gamma<0.15$, in consistent with the theoretic value 0.2 given by Sumi. ${ }^{43}$ When $\gamma>0.5$, $\kappa_{\mathrm{el}}$ deviates from the linear dependence on $\gamma$ and approaches unity for $\gamma>1$, showing the $\gamma$-dependence of $\kappa$ as theoretically predicated. ${ }^{2,43}$ Therefore, the experimental results are generally in accordance with theoretic results, but give a narrower and more precise window of $\gamma$ in the correlations between $\kappa_{\mathrm{el}}$ and $\gamma$ in the different regimes. For the three systems in $\left[\mathbf{E E}^{\prime}-(\mathbf{p h})_{3^{-}}\right.$ $\left.\mathbf{E E}^{\prime}\right]^{+},\left[\mathbf{O O}-(\mathbf{p h})_{3}-\mathbf{O O}\right]^{+}$has a Jortner adiabatic parameter $\kappa_{\mathrm{A}}=0.5(<1),{ }^{15,44}$ while for the other two, $\kappa_{\mathrm{A}}=6-8(>1)$, showing the agreement between the two criteria in defining the two limits.

For systems with $P_{0}<\kappa_{\mathrm{el}}$, involvement of multiple passages in thermal ET reactions is anticipated. For the intermediate systems, it is assumed that two channels, the single passage and the first multiple passage, operate for nonadiabatic transition, as described by Fig. 3B. In the first channel, the electron makes a transition from the reactant to the product state through the crossing point, giving the probability $P_{0}$. In the second channel, the electron passes the crossing point three time to complete the reaction. The first (step 1) and third (step 3) crossing take place on the reactant and product diabatic PESs, respectively, which have the same probability, $\left(1-P_{0}\right)$. Electron hopes 
from the reactant to the product PES through the second transition (step 2) with the same probability as the first channel $\left(P_{0}\right)$. This multiple passage gives the transition probability of $\left(1-P_{0}\right) P_{0}\left(1-P_{0}\right) .^{2}$ For $\left[\mathbf{O S}-(\mathbf{p h})_{3}-\mathbf{O S}\right]^{+}$and $\left[\mathbf{S S}-(\mathbf{p h})_{3}-\mathbf{S S}\right]^{+}$, a transition probability of 0.15 and 0.14 is obtained from the multiple passage, respectively. The total probabilities in this two-channel scheme, ca. 0.47 and 0.55 , are close to the overall transmission probabilities $\left(\kappa_{\mathrm{el}}\right) 0.48$ and 0.58(Table 1), respectively. This means that for these two systems, $98 \%$ and $95 \%$ of the successful nonadiabatic hopping events proceed through the first and second channels, with the first channel playing the dominant role. For $\left[\mathbf{O S}-(\mathbf{p h})_{3}-\mathbf{O S}\right]^{+}$, the overall transition probability is slightly small, in comparison with [SS-(ph) $\left.)_{3}-\mathbf{S S}\right]^{+}$, but the multiple passage contribution is relatively large due to 10 the increased nonadiabaticity. It is believed that this two-channel operation can be the typical behavior for thermal ET systems on the adiabatic-nonadiabatic borderline. For [OO-(ph) $\left.\mathbf{3}_{3}-\mathbf{O O}\right]^{+}$, the single and multiple passages are nearly equally important, each of which contributes a transition probability of $\sim 0.07$. Evidently, this system has entered the nonadiabatic regime through the intermediate. The small $\kappa_{\mathrm{el}}(0.14)$ visulizes the failure of thermal ET through nonadiabatic transtion due to the high activation erengy, $\Delta \mathrm{G}^{*} \approx \lambda / 4$ (Table 1 ). However, this does not mean no electron self-exchagne occurring between the donor and acceptor. For this long-bridge, weakly coupled system, the nonadiabatic ET may occur through optical transiton, ${ }^{9}$ the highly energetic pathway at the same ET rate as for the thermal ET pathway. ${ }^{21}$ The multiple trajectory model, developed based on the Fermi Golden rule, ${ }^{31}$ is the core of the quantum mechanism for nonadiabatic reactions. ${ }^{1,2,43}$ However, trajectory analysis of ET in experimental systems is rarely seen.

The LZ model was developed to deal with nonadiabatic coupling in the vicinity of the avoied crossing where $\Delta \mathrm{G}^{*} \gg 2 H_{\mathrm{ab}}$. The theory cannot tell what happens if the kinetic energy is comparable to the interaction energy. ${ }^{2,12}$ This is the case represented by $[\mathbf{E E} \text { '-ph-EE' }]^{+}$, for which $\Delta \mathrm{G}^{*}<2 \mathrm{H}_{\mathrm{ab}}$. Supprisingly, even for the mostly strongly coupled [SS-ph-SS] $]^{+}$, with the activation energy $\left(\Delta \mathrm{G}^{*}=79 \mathrm{~cm}^{-1}\right)$ much smaller than the coupling energy $\left(H_{\mathrm{ab}}=864 \mathrm{~cm}^{-1}\right)$, the thermal ET can be well described by the LZ parameters, that is, $\gamma=5.32(>1), \mathrm{P}_{0}=1$ and $\kappa_{\mathrm{el}}=1$. Moreover, theoretically, application of the LZ model is limited by the requirment of narrow avoded crossing, that is, that the minimal spacing between the adiabatic PESs at the avoided region should be much smaller than the spacing far from the coupling region. ${ }^{12}$ Again, taking [SS-ph-SS] ${ }^{+}$as an example, 
the separations between the surfaces $V_{1}$ and $V_{2}$ at the reactant equilibrium, i.e., $E_{\mathrm{IT}}=2640 \mathrm{~cm}^{-1}$ and at the transtion state, i.e., $2 H_{\mathrm{ab}}=1728 \mathrm{~cm}^{-1}$, are in the same order of magnitude, which breakdowns the narrow avoided-crossing approxiamtion. Collectively, this study provides the first precise scaling picture showing system transition from the adiabatic to nonadaibatic ET limits through the intermediate, which demostrates the validity of the Landau-Zener model. The experimental results extend the theoretic limitation in application of the LZ formula, and offers the details of the energetics, dyanamics and kinttics for system crossover.

\section{References and Notes:}

1. Newton, M. D. \& Sutin, N. Electron transfer reaction transfer in condensed phases. Ann. Rev. Phys. Chem. 35, 437-480 (1984).

2. Balzani, V. Electron transfer in chemistry (Wiley-VCH, Weinheim, Germany, 2001).

3. Marcus, R. A. On the theory of oxidation-reduction reactions involving electron transfer. $J$. Chem. Phys. 24, 966-978 (1956).

4. Closs, G. L. \& Miller, J. R. Intramolecular long-distance electron transfer in organic molecules. Science 240, 440-446 (1988).

5. Marcus, R. A. \& Sutin, N. Electron transfers in chemistry and biology. Biochim. Biophys. Acta 811, 265-322 (1985).

6. Page, C. C., Moser, C. C., Chen, X. \& Dutton, P. L. Natural engineering principles of electron tunnelling in biological oxidation-reduction. Nature, 402, 47-52 (1999).

7. Choi, S. H., Kim, B. \& Frisbie, C. D. Electrical resistance of long conjugated molecular wires. Science 320, 1482-1486 (2008).

8. Blankenship, R. E. et al. Comparing photosynthetic and photovoltaic efficiencies and recognizing the potential for improvement. Science 332, 805-809 (2011).

9. Creutz, C. Prog. Inorg. Chem. Mixed-valence-complexes-of $\mathrm{d}^{5} \mathrm{~d}^{6}$-metal-centers. 30, 1-73 (1983).

10. Landau, L. D. Phys. Z. Sowjetunion 1, 88-98 (1932). 
11. Zener, C. Non-adiabatic crossing of energy levels. Proc. R. Soc. London A 137, 696-702 (1932).

12. Nikitin, E. E. NONADIABATIC TRANSITIONS what we learned from old masters and how much we owe them. Annu. Rev. Phys. Chem. 50, 1-21 (1999).

13. Newton, M. D. Quantum chemical probes of electron-transfer kinetics: The nature of donoracceptor interactions. Chem. Rev. 91, 767-792 (1991).

14. Zusman, L. D. Outer-sphere electron transfer in polar solvents. Chem. Phys. 49, 295-304 (1980).

15. Rips, I. \& Jortner, J. Dynamic solvent effects on outer-sphere electron transfer. J. Chern. Phys. 87, 2090-2104 (1987).

16. Mühlbacher, L. \& Egger, R. Crossover from nonadiabatic to adiabatic electron transfer reactions Multilevel blocking Monte Carlo simulations. J. Chem. Phys. 118, 179-191 (2003).

17. Ito, T., Hamaguchi, T., Nagino, H., Yamaguchi, T., Washington, J. \& Kubiak, C. P. Effects of Rapid Intramolecular Electron Transfer on Vibrational Spectra. Science 277, 660-663 (1997).

18. Creutz, C. \& Taube, H. Direct approach to measuring the Franck-Condon barrier to electron transfer between metal ions. J. Am. Chem. Soc. 91, 3988-3989 (1969).

19. Hush, N. S. Intervalencetransfer-absorption-part-2-theoretical-consideration. Prog. Inorg. Chem. 8, 391-444 (1967).

20. Nelsen, S. F., Ismagilov, R. F. \& Trieber, D. A. Adiabatic electron transfer comparison of modified theory with experiment. II, Science 278, 846-849 (1997).

21. Elliott, C. M., Derr, D. L., Matyushov, D. V. \& Newton, M. D. Direct experimental comparison of the theories of thermal and optical electron-transfer studies of a mixed-valence dinuclear iron polypyridyl complex. J. Am. Chem. Soc. 120, 11714-11726 (1998).

22. Schäffer, L. J. \& Taube, H. Intramolecular electron transfer through isomeric forms of dlcyanobenzene. J. Phys. Chem. 90, 3669-3673 (1986).

23. Distefano, A. J., Wishart, J. F. \& Isied, S. S. Convergence of spectroscopic and kinetic electron transfer parameters for mixed-valence binuclear dipyridylamide ruthenium ammine complexes. Coord. Chem Rev. 249, 507-516 (2005). 
24. Demadis, K. D., Hartshorn, C. M. \& Meyer, T. J. The localized-to-delocalized transition in mixed-valence chemistry. Chem. Rev. 101, 2655-2685 (2001).

25. Creutz, C. \& Taube, H. Binuclear complexes of ruthenium ammines. J. Am. Chem. Soc. 95, 1086-1094 (1973).

26. Cotton, F. A. \& Nocera. D. G. The whole story of the two-electron bond, with the $\delta$ bond as a paradigm. Acc. Chem. Res. 33, 483-490 (2000).

27. Liu, C. Y., Xiao, X., Meng, M., Zhang, Y. \& Han, M. J. Spectroscopic study of $\delta$ electron transfer between two covalently bonded dimolybdenum units via a conjugated bridge adequate complex models to test the existing theories for electronic coupling. J. Phys. Chem. C 117, 19859-19865 (2013).

28. Xiao, X., Meng, M., Lei, H. \& Liu, C. Y. Electronic coupling and electron transfer between two dimolybdenum units spaced by a biphenylene group. J. Phys. Chem. C 118, 8308-8315 (2014).

29. Tan, Y. N., Cheng, T., Meng, M., Zhang, Y. Y., Liu, C. Y., Sun, M. F., Zhang, Y. \& Low, P. J. Optical behaviors and electronic properties of Mo2-Mo2 mixed-valence complexes within or beyond the Class III regime testing the limits of the two-state model. J. Phys. Chem. C 121, 27860-27873 (2017).

30. Isied, S. S., Vassilian, A. \& Wishart, J. F. The distance dependence of intramolecular electron-transfer rates importance of the nuclear factor. J. Am. Chem. Soc. 110, 635-637 (1988).

31. Barbara, P. F., Meyer, T, J. \& Ratner, M. A. Contemporary issues in electron transfer research. J. Phys. Chem. 100, 13148-13168 (1996).

32. Xiao, X., Liu, C. Y., He, Q., Han, M. J., Meng, M., Lei, H. \& Lu, X. Control of the charge distribution and modulation of the Class II-III transition in weakly coupled Mo2-Mo2 systems. Inorg. Chem. 52, 12624-12633 (2013).

33. McConnell, H. M. Intramolecular charge transfer in aromatic free radicals. J. Chem. Phys. 35, 508-515 (1961). 
34. Rosokha, S. V., Sun, D. L. \& Kochi, J. K. Conformation, distance, and connectivity effects on intramolecular electron transfer between phenylene-bridged aromatic redox centers. J. Phys. Chem. A, 106, 2283-2292 (2002).

35. Brunschwig, B. S., Creutz, C. \& Sutin, N. Optical transitions of symmetrical mixed-valence systems in the Class II-III transition regime. Chem. Soc. Rev. 31, 168-184 (2002).

36. Creutz, C., Newton, M. D. \& Sutin, N. Metal-lingad and metal-metal coupling elements. $J$. Photochem. Photobiol. A 82, 47-59 (1994).

37. Toutounji, M. M. \& Ratner, M. A. Testing the condon approximation for electron transfer via the mulliken-hush model. J. Phys. Chem. A, 104, 8566-8569 (2000)

38. Robin, M. B. \& Day, P. Mixed valence chemistry-A survey and classification. Adv. Inorg. Chem. Radiochem 9, 247-422 (1967).

39. Brunschwig, B. S. \& Sutin, N. Energy surfaces, reorganization energies, and coupling elements in electron transfer. Coord. Chem. Rev. 187, 233-254 (1999).

40. Lambert, C. \& Nçll, G. The Class II/III transition in triarylamine redox systems. J. Am. Chem. Soc. 121, 8434-8442 (1999).

41. Levich, V. G. Present state of the theory of oxidation-reduction in solution (bulk and electrode reactions). Adv. Electrochem. Electrochem. Eng. 4, 249-371 (1966).

42. Cheng, T. et al. Efficient electron transfer across hydrogen bond interfaces by protoncoupled and -uncoupled pathways. Nat. Commun. 10, 1531 (2019).

43. Sumi, H. Energy transfer between localized electronic states -From non-adiabatic to adiabatic hopping limits- J. Phys. Soc. Jpn. 49, 1701-1712 (1980).

44. The Jortner adiabatic parameter $\left(\kappa_{\mathrm{A}}\right)$ is calculated from $\kappa_{\mathrm{A}}=\frac{4 \pi H_{A B}^{2}\langle\tau\rangle}{\mathrm{h} \lambda_{\mathrm{o}}}$ (ref. 15) using $\tau=1$ ps.

\section{Acknowledgments:}

We are greatly thankful to the National Natural Science Foundation of China (No. 21971088, 21371074), Natural Science Foundation of Guangdong Province (No. 2018A030313894), Jinan 
University, and Fundamental Research Funds for the Central Universities for the financial support.

\section{Author contributions:}

C.Y.L. conceived this project and designed the experiments and worked on the manuscript. G.Y.Z. and Y.Q. carried out the major experimental work. M.M. completed the data collection and analysis of the X-ray crystal structures. M.M. and M.S. participated in data analysis and manuscript preparation, H.G., X.C., X.X., T.C. and M.J.H were involved in experimental investigation.

$\dagger$ These authors are equally contributed.

\section{Competing interests:}

The authors declare no conflict of interest.

\section{Data and materials availability:}

The X-ray crystallographic data of [OS-(ph) $\left.)_{3}-\mathbf{O S}\right]$ reported in this study have been deposited at the Cambridge Crystallographic Data Centre (CCDC), under deposition number CCDC 2004426. These data can be obtained free of charge from The Cambridge Crystallographic Data Centre via www.ccdc.cam.ac.uk/data request/cif. The data that support the findings of this study are available from the corresponding authors upon reasonable request.

All the data supporting the findings of this study are available from the corresponding author upon reasonable request. 
Figures

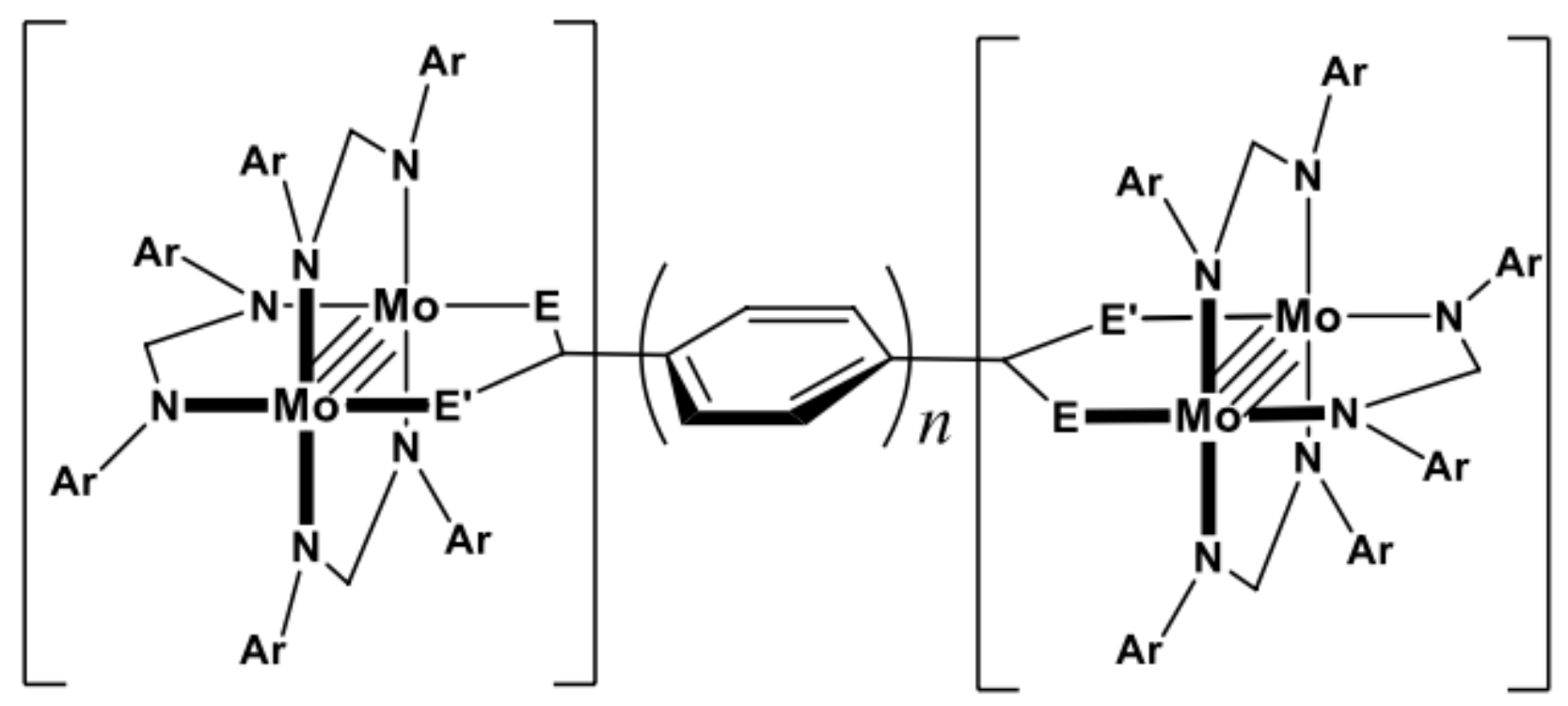

$\left[\mathrm{Mo}_{2}\right]-(\mathrm{ph})_{n}-\left[\mathrm{Mo}_{2}\right]$

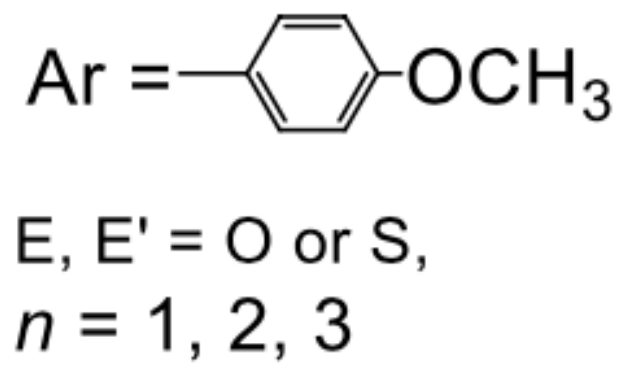

[EE'-(ph) $)_{n}$-EE']:

1. $\left[00-(\mathrm{ph})_{n}-\mathrm{OO}\right]$

2. [OS-(ph) $\left.)_{n}-\mathrm{OS}\right]$

3. [SS-(ph) $\left.)_{n}-\mathbf{S S}\right]$

Figure 1

A Molecular scaffold for the complexes under investigation. The three series of [Mo2]-(ph)n-[Mo2] complexes are differentiated by the [Mo2] complex units due to O/S alternation of the chelating atoms ( $E$ and $\left.E^{\prime}\right)$. Each series consists of three complexes with different (poly)phenylene bridges (phn, $n=1-3$ ). 

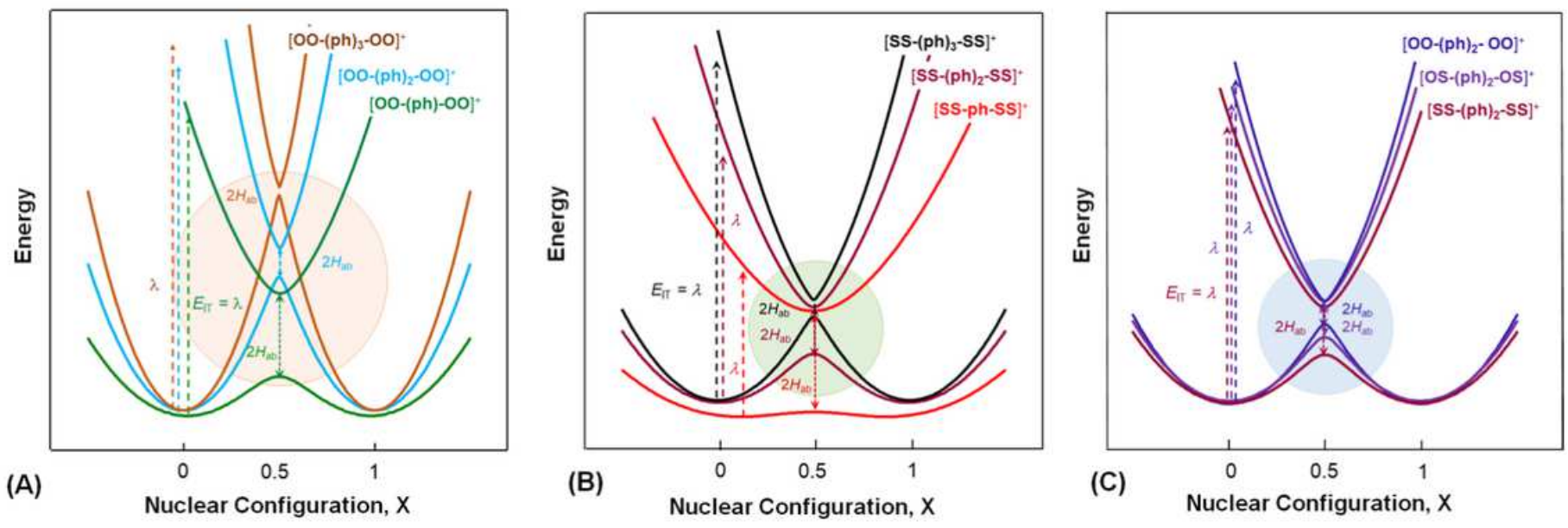

Figure 2

Diagrams of the adiabatic potential energy surfaces for the mixed-valence $\{[\mathrm{Mo} 2]-(\mathrm{ph}) \mathrm{n}-[\mathrm{Mo} 2]\}+$ derived from eq 5 based on the optically determined $\lambda$ and Hab. (A), Series [00-(ph)n-00]+ $(n=1-3)$. (B), Series [SS-(ph)n-SS]+ $(n=1-3) .(C)$, Series $\left[E E^{\prime}-(p h) 2-E E^{\prime}\right]+\left(E, E^{\prime}=0\right.$ or $\left.S\right)$. For each of the PES diagrams, the avoided crossing area is highlighted.

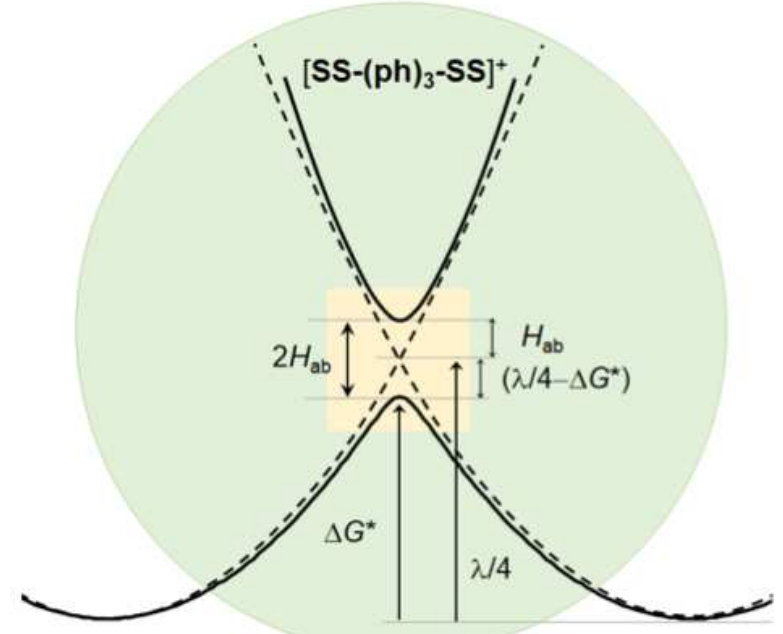

(A)

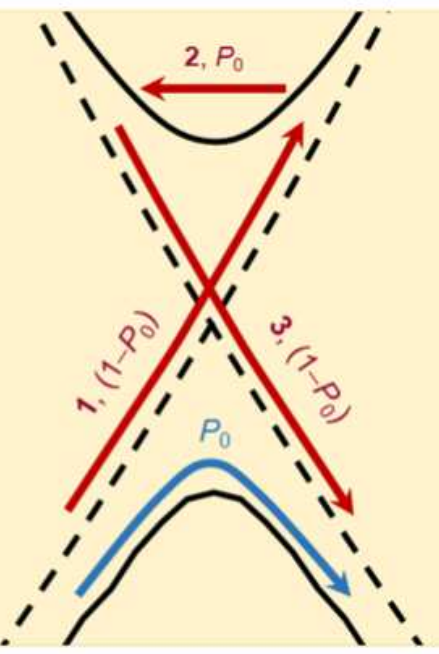

(B)

\section{Figure 3}

Schematic representations of the energetic features of the crossing region and nonadiabatic transition channels for the transient systems. (A), The diabatic (dashed line) and adiabatic (solid line) potential energy surfaces in the reactant and product equilibriums and the transition state for [SS-(ph)3-SS]+. (B), The first (cyan) and second (red) channels of nonadiabatic transition in intermediate system [SS-(ph)3SS]+. The potential surfaces in (A) and (B) result from zooming in of the avoided crossing area in Fig. 2B. Similar results are expected for [OS-(ph)3-OS]+ from the similar $\lambda$ and Hab data. 

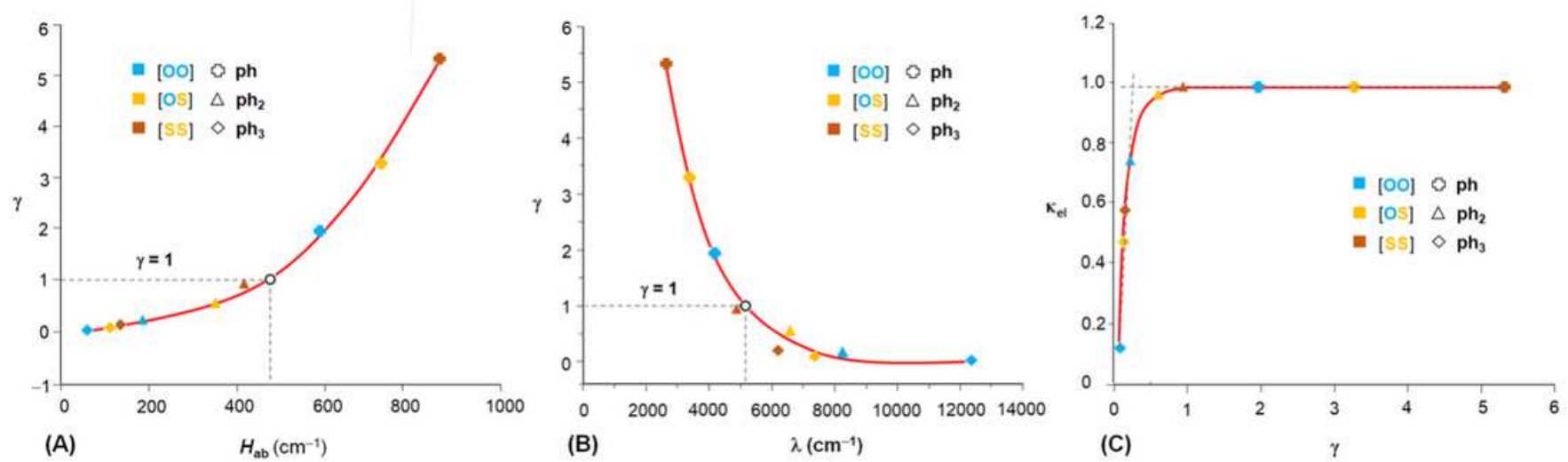

Figure 4

Impacts of the electronic and nuclear factors on the Landau-Zener parameters $₫$ and $囚 e$ in systems [EE'-

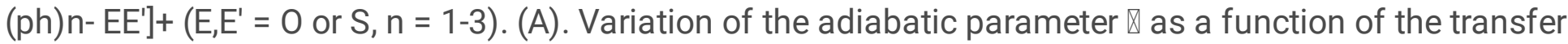
integral (Hab). (B) Variation of the transmission coefficient \el as a function of reorganization energy $(\lambda)$. (C). 囚-dependence of 『el. Color codes for [EE'-(ph)n- EE']+: blue for [OO-(ph)n-0O]+, yellow for [OS-(ph)nOS]+ and brown for [SS-(ph)n-SS]+. The ph, ph2 and ph3 bridges are represented by cross, triangle and square, respectively.

\section{Supplementary Files}

This is a list of supplementary files associated with this preprint. Click to download.

- naturecommlzSI.doc

- 3phOS.cif

- checkcif.pdf 